\title{
New EPMA Applications Utilizing Novel High-current, High-spatial Resolution Field Emission Optics
}

Christopher Macey $^{1}$ and Takeshi Miyamoto ${ }^{2}$

${ }^{1}$ Shimadzu Scientific Instruments, Inc., Columbia, Maryland, United States, ${ }^{2}$ Shimadzu Corportion, Kyoto, Kyoto, Japan

In this paper we describe the Shimadzu EPMA-8050G, a novel field-emission EPMA that utilizes a patented optics design and x-ray take off angles as high as 52.5 degrees to obtain high spatial resolution at high beam currents. Beam conditions of 20nm@10nA and maximum $3 \mu \mathrm{A}$ beam current are achievable.

This improved design allows EPMA elemental analysis to be utilized in new applications that require both high spatial resolution and high elemental sensitivity.

The probe size, which is the main factor of spatial resolution, increases with increasing beam current. At a certain current threshold, Ith, there is an increase in the rate at which the probe size increases with beam current. The factors which influence this threshold were investigated and optimized.

A derived formula (1) was used for this research [1] [2].

Ith $=4^{2 / 3} \pi(k B T / e)^{1 / 3} f^{8 / 3} j s / \operatorname{Csg}^{2 / 3} \operatorname{Vext}^{1 / 3}(1)$

\section{kB: Boltzmann constant, T: emitter temperature, e: electronic charge}

$f g$ : electron gun focal length, $j s$ : cathode current density

Csg: gun spherical aberration, Vext: extraction voltage

According to formula (1), Ith the threshold beam current can be increased by the decrease of Csg and/or the increase of fg. In designing the EPMA-8050G, a decrease of Csg was achieved by optimizing the shape of magnetic focusing lens [3]. The value of fg is dependent on the nose shape of the emitter. Decreased fg was achieved by the choice of the shape of the emitter.

This technology realizes microanalysis examples illustrating both high elemental sensitivity and high spatial resolution are provided.

Fig.1 (right) shows the relationship between beam current and probe size achieved with the EPMA-8050G. The data show the increased current threshold. The EPMA-8050G is shown in Fig.1 (left).

Fig.1 (under) shows the difference in secondary electron image quality of Sn balls on carbon when the irradiation current is changed to $10 \mathrm{nA}, 100 \mathrm{nA}$, and $1 \mu \mathrm{A}$. These have been realized by the installation of new Schottky emitter, electron optical system and an ultra-high vacuum evacuation system. These new technologies can finely focus the electron beam to this point even at the time of irradiation with a large current, thus maintaining good beam characteristics during elemental analysis.

The electron optical system has a proprietary configuration and control method (Japan Patent No. 4595778). The condenser lens is set as close as possible to the electron gun. Crossover is formed not with the condenser lens but with an iris lens, with the objective aperture arranged at the same position. While this is a simple lens configuration, a large current can be obtained. At the same time, the angular aperture can be optimally configured under all current conditions, minimizing the electron beam diameter. Naturally, there is no need to replace the objective aperture. 
In addition, the high X-ray take-off angle of $52.5^{\circ}$, which is the concept of the EPMA made by Shimadzu Corporation, makes it possible to perform better analysis on a variety of samples, such as unpolished and curved samples.

Fig.2 show analyses of high spatial resolution elemental mapping respectively. Fig.2a-c (left) shows data obtained by mapping analysis of a region containing much Ag in the lead-free solder. In the backscattering electron image, the difference in contrast between the red-round particles and the yellow-round particles is small and cannot be distinguished, but the X-ray image confirms that the red-round particles are $\mathrm{Ag}$ and the yellow-round particles are $\mathrm{Cu}$. It can also be seen that particles with a diameter of about $100 \mathrm{~nm}$ can be identified by X-ray images. Also, Fig.2a-b (right) shows the ultrafine particles in lung tissue

Since the EPMA-8050G can irradiate a large current of up to $3 \mu \mathrm{A}$, it is possible to detect an extremely trace element with high sensitivity. Fig.2 (under) shows the result of mapping analysis of Si contained in stainless steel at about $1 \%$ at different irradiation current values. It can be seen that the larger the irradiation current value, the stronger the signal intensity and the clearer the region containing Si.

By various new technologies, the EPMA-8050 has achieved the world's highest performance as an EPMA. In addition, by combining technology cultivated over a long history, applications that have been difficult until now can be realized, and they are expected to be used in various fields and applications in the future.

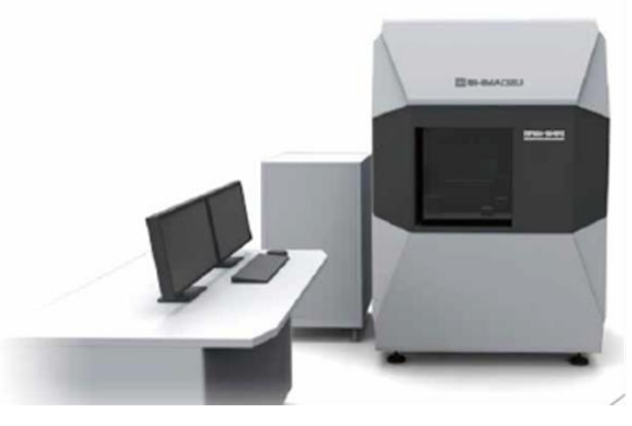

$$
\text { 10nA }
$$

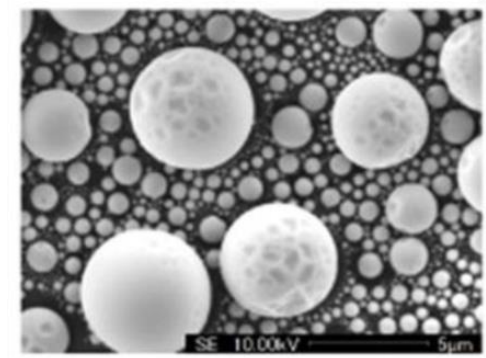

Figure 1. Left; FE-EPMA EPMA-8050G / Right; Relationship between beam current and probe size / Under; SE image under mapping beam current condition by EPMA-8050G 


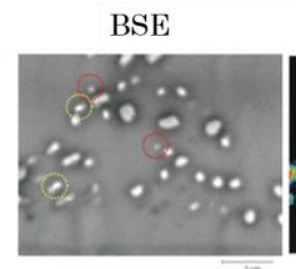

(a)

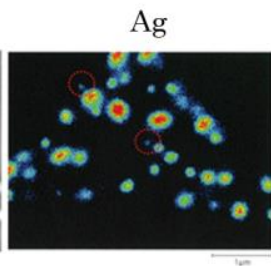

(b)

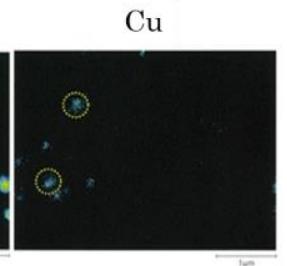

(c)

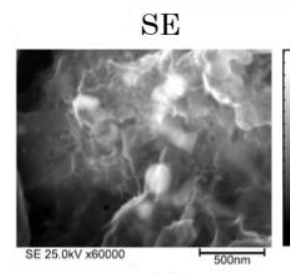

(a)

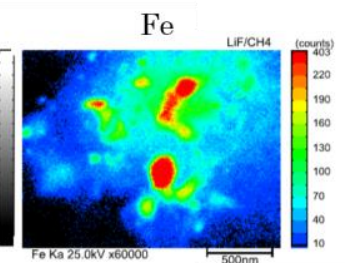

(b)
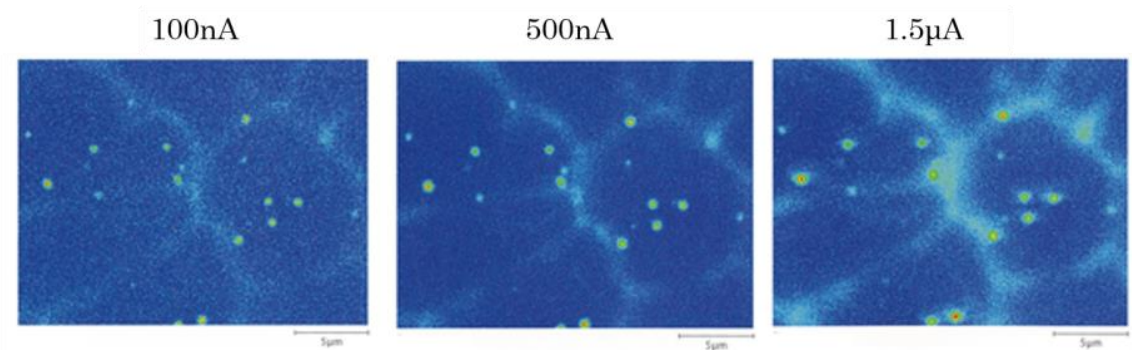

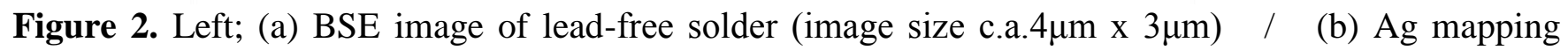
image / (c) Cu mapping image / Right; (a) SE image of ultrafine particles in lung tissue / (b) Fe mapping image (image size c.a. 2um x 1.5um) / Under; Si mapping image of $1 \% \mathrm{Si}$ in stainless steel

\section{References}

[1] S. Fujita, T.R.C. Wells, W. Ushio, H. Ssto and H.M. El-Gomati: Enhanced angular current intensity from Schottky emitters, J. Microscopy, 239(3),215(2010)

[2] S. Fujita and H. Shimoyama: A new evaluation method of electron optical performance of high beam current probe forming systems, J. Electron Microsc., 54(5), 413(2005)

[3] H. Shimoyama, K. Nimura, M. Suzuki and S. Maruse: Magnetic-Field-Superposed Electron Gun with a Point Cathode, J. Electron Microsc., 32(2), 99(1983) 